\title{
Performance Evaluation at Different Bit Rates of Star, Tree and Mesh Topology using Raman-EDFA Hybrid Optical Amplifier
}

\author{
Sanjeev Dewra ${ }^{1}$, Rajpreet Singh $^{2}$
}

\begin{abstract}
Performance at different bit rates of phase modulated signals for star, tree and mesh optical network topologies is evaluated using Raman-EDFA hybrid optical amplifier. Consideration of BER is made to investigate the number of users supported. Evaluation of the number of users supported is

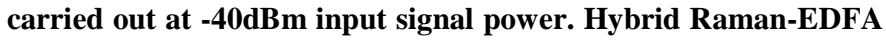
amplifier is placed as a post amplifier. In all these three topologies, the signals are generated at $90^{\circ}$ phase deviation. The channel spacing is taken as $0.1 \mathrm{THz}$ with $1 \mathrm{MHz}$ line width. Mesh topology supports more number of users than other topologies at $10 \mathrm{Gbps}$ and $20 \mathrm{Gbps}$. At the bit rate of $10 \mathrm{Gbps}$ within acceptable BER range, Mesh topology supports 8 number of users whereas star topology supports 5 users and tree topology supports 4 users. At 20Gbps within acceptable BER range, Mesh topology supports 6 users whereas tree topology supports 4 users and star topology supports 2 users. No topology is able to support any user at 30 Gbps.
\end{abstract}

Keywords - hybrid optical amplifier, mesh topology, star topology, tree topology.

\section{Introduction}

From the early times, It remained a need to transmit data at high bit rates. Performance of the transmission degrades at higher bit rates due to the nonlinear nature of the optical fiber. In a communication system, the receiver side BER may be affected by transmission channel noise, interference, distortion, bit synchronization problems, attenuation, wireless multipath fading etc.

Hybrid optical amplifiers perform better as compared to single amplifier. EDFA amplifier gives terrific performance by the data rate amplification because of its large gain bandwidth

Dr. Sanjeev Dewra

Shaheed Bhagat Singh State Technical Campus India

Rajpreet Singh

Shaheed Bhagat Singh State Technical Campus India
[1]. Reduction in non linear penalty with enhanced noise figure is given by Raman amplifier [2]. Raman-EDFA hybrid optical amplifier performs better in terms of increased output power and reduced BER than in EDFA, SOA and hybrid EDFA-SOA [3]. Singh [4] revealed post amplifier scheme of Hybrid Raman-EDFA gives better results in terms of reduced BER and increased output power than in other schemes. Compensation in range loss and chromatic dispersion is balanced by Hybrid Raman-EDFA amplifier. For long distance transmission WDM can be preferred [5].

In star optical network, links are created between couplers in order to carry information from transmitters to receivers. Distribution of output power takes place at receiving end. Y.N. Singh [6] revealed that adding SOA can bring increment in the number of users. At $-30 \mathrm{dBm}$, only four users are supported at bit rate of $1 \mathrm{Gbps}$. He revealed that efficiency can be increased by using SOA as preamplifier.

In Tree optical network, substitution of optical star over bus topology takes place. It is a combination of star and bus topology. Scalability is provided by this network i.e. large number of users can be attached to single node with the help of secondary nodes. Tree networks are used widely over globe. M.Gerla,et al [7] demonstrated that number of users over this network is product of branches and users per branch. Y.N. Singh [8], reported that tree network supports large number of users at $1 \mathrm{Gbps}$.

In Mesh Optical network, nodes are interconnected. It provides secure as well as reliable transmission. It provides several advantages like enabling of more general routing schemes, more flexible traffic engineering, simplification of network operations and management. Mesh network can be applied physically at physical layers and logically at logical layers [9].

In this paper, we proposed star, tree and mesh optical network topologies in addition of optimized Hybrid RamanEDFA amplifiers. The performance of these topologies is evaluated for Pulse Modulated signals at different bit rates for a given number of users in terms of Quality Factor and Received output power at $-40 \mathrm{dBm}$ input signal power. The results in this paper are coincidence with previous results [6] where it was observed that topologies using SOAs is capable to carry out the users at bit rate of $1 \mathrm{Gbps}$ with signal input signal power of $-30 \mathrm{dBm}$ for star topology. In this paper, the work is extended using Raman-EDFA hybrid optical amplifier and compared the results for these three topologies in terms of least input signal power of $-40 \mathrm{dBm}$ at different bit rates. 
Rest of the paper is presented as follows. In section II, transmitter and amplifier used is provided. In section III, design of the topologies is provided, section IV provided simulation result and discussion and section $\mathrm{V}$ provided Conclusion.

\section{Transmitter and Amplifier}

\section{A. Transmitter}

At transmitter side, CW laser is used to provide continuous wave. The PRBS or Pseudo-Random Bit Sequence generator is used to generate pseudorandom bits. NRZ is for Non Return to Zero pulse generation. The signal generated is modulated through second order of phase modulator.

\section{B. Amplifier}

The amplifier used in setup is Hybrid Raman-EDFA amplifier i.e. EDFA is placed next to Raman Amplifier. For designing Raman model, average power model is preferred as it reduces the computational time required to solve the Raman Amplifier differential equations by simplifying the equations. External backward pumping is provided to Raman Amplifier. In EDFA model, amplified spontaneous emission (ASE) is included. This combination of amplifiers is used as a post amplifier in all the three topologies.

\section{Topologies Using Hybrid Raman-EDFA Amplifier}

\section{A. Star Topology}

In forming star optical network, $2 \times 2$ optical couplers are used to form $U_{n} \times U_{n}$ network [10]. Star network using hybrid Raman-EDFA amplifier is shown in Figure 1. In star network, information is transmitted from the transmitter at different bit

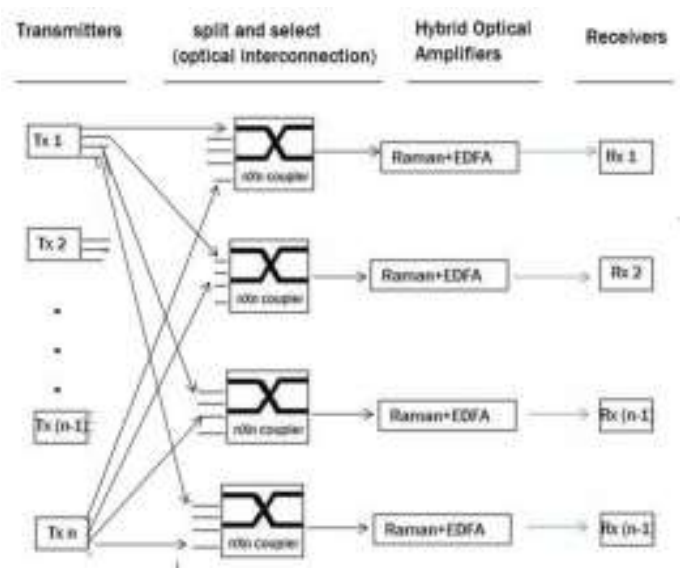

Figure 1 Star Topology rates and by passing through the different paths reaches at the

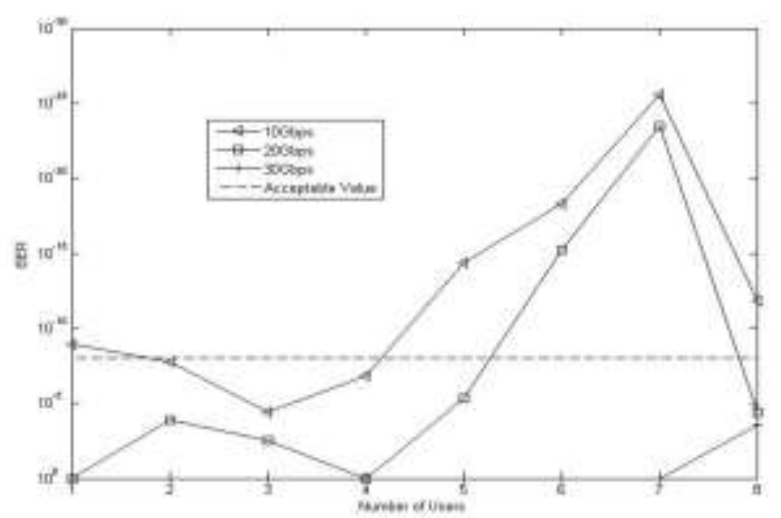

Figure 2 Bit Error Rate (BER) as a function of bit rate for different number of users in Star topology using Hybrid Raman-EDFA amplifier

receiver side. The distance taken between the links is taken as $1 \mathrm{Km}$. Before reaching at receiver the signal is made to pass through hybrid Raman-EDFA amplifier. The Bit Error Rate(BER) for different number of users at different bit rates is represented in Figure 2. It is observed that with the increment in bit rate, the Bit Error Rate Increases. It is due to material dispersion i.e. dependence of frequency on fiber index which leads to broadening of pulse [9]. The estimation of the effect of dispersion on bit rate is given as

$$
B \Delta T<1
$$

Where $\mathrm{B}$ is bit rate and $\Delta T$ is delay in time and is given as:

$$
\Delta T=D L \Delta \lambda
$$

Where $\mathrm{D}$ is dispersion parameter, $\mathrm{L}$ is length of the fiber and $\Delta \lambda$ is range of wavelength emitted by optical source. Putting the value of $\Delta T$ from eq. (2) to eq. (1):

$$
B L|D| \Delta \lambda<1
$$

\section{B. Tree Topology}

In forming tree optical network, folded bus and star plays

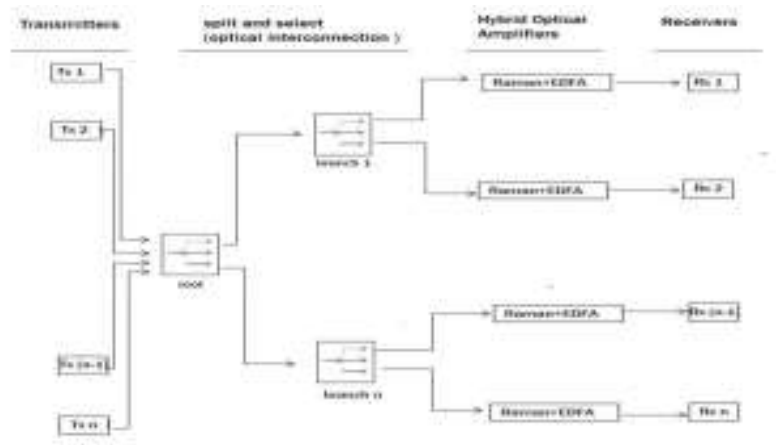

Figure 3 Tree topology 


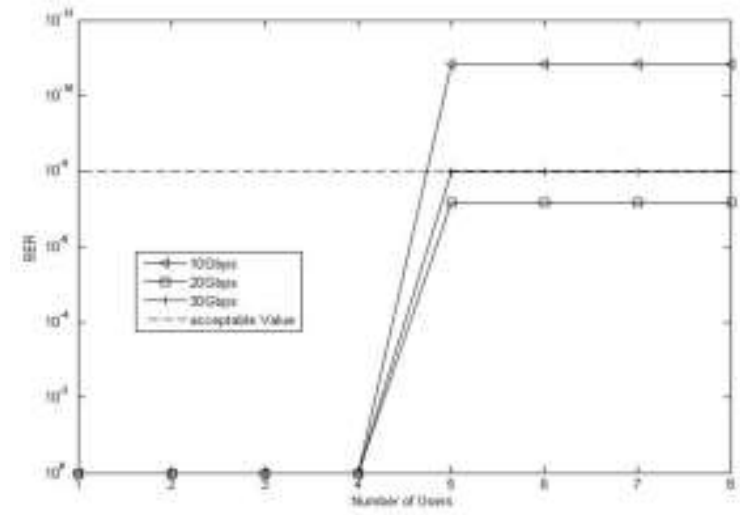

Figure 4 Bit Error Rate (BER) as a function of bit rate for different number of users in Tree topology using Hybrid Raman-EDFA amplifier

role. User over this network is given as product of branches and users per branch [11]. Tree network using Hybrid RamanEDFA amplifier is shown in Figure 3. Information is transmitted from the transmitters at different bit rates and is made to pass through different paths through which it reaches at receiving end. Before reaching at receiver, the signals are made to pass through the Hybrid Raman-EDFA amplifier. The Bit Error Rate (BER) for different number of users at different bit rates is represented in Figure 4. It is observed that with the increment in bit rate, the Bit Error Rate Increases. It is due to material dispersion [9].

\section{Mesh Topology}

There are two type of mesh topologies i.e. partial mesh topology and complete mesh topology. In this setup, complete mesh topology is used in which all the nodes are

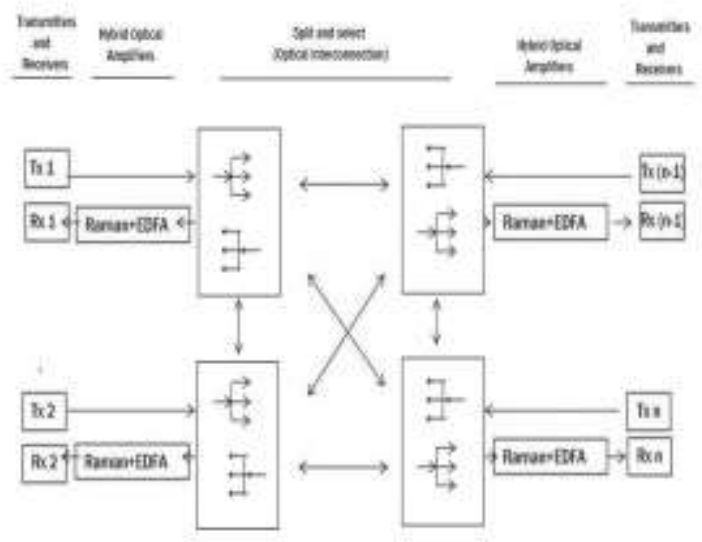

Figure 5 Mesh Topology

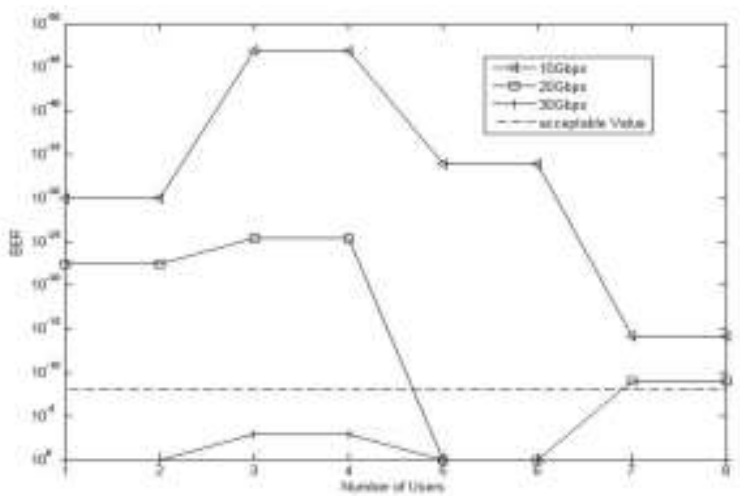

Figure 6 Bit Error Rate (BER) as a function of bit rate for different number of users in Mesh topology using Hybrid Raman-EDFA amplifier

interconnected. The model is shown in figure 5. During the transmission, the power is spitted among different users at different nodes whereas while receipting, the power from different nodes is combined making a node to transmit and receive the data. Before reaching to the receiver of the nodes, the signals are made to pass through the Hybrid RamanEDFA. The Bit Error Rate (BER) for different number of users at different bit rates is represented in Figure 6. It is observed that with the increment in bit rate, the Bit Error Rate Increases. It is due to material dispersion [9].

\section{Results and Discussion}

The simulation is performed at signal input power of $40 \mathrm{dBm}$. In these entire three star, tree and mesh optical network topologies, Raman-EDFA optical amplifier is used as post amplifier. The results in this paper are coincidence with previous results [6] where it was observed that topologies using SOAs is capable to carry out the users at bit rate of $1 \mathrm{Gbps}$ with signal input signal power of $-30 \mathrm{dBm}$ for star topology. In this paper, the work is extended using RamanEDFA hybrid optical amplifier and compared the results for these three topologies in terms of least input signal power of $40 \mathrm{dBm}$ at different bit rates. The parameters of HOA are given in Table I. The signals for the simulation are generated in pseudorandom manner at order 2 with phase modulation.

\begin{tabular}{|c|c|}
\hline \multicolumn{2}{|c|}{ EDFA Parameters } \\
\hline Length & $5 \mathrm{~m}$ \\
\hline Pump(F) Power & $100 \mathrm{~mW}$ \\
\hline Pump(B) Power & $0 \mathrm{~mW}$ \\
\hline $\begin{array}{c}\text { Pump(F) } \\
\text { Wavelength }\end{array}$ & $980 \mathrm{~nm}$ \\
\hline $\begin{array}{c}\text { Pump(B) } \\
\text { Wavelength }\end{array}$ & $980 \mathrm{~nm}$ \\
\hline Core Radius & $2.2 \mathrm{~m}$ \\
\hline Er Doping Radius & $2.2 \mathrm{~m}$ \\
\hline
\end{tabular}

\begin{tabular}{|c|c|}
\hline \multicolumn{2}{|c|}{ Raman Parameters } \\
\hline Length & $10 \mathrm{Km}$ \\
\hline Attenuation & $0.2 \mathrm{~dB} / \mathrm{Km}$ \\
\hline Temperature & $300 \mathrm{~K}$ \\
\hline Pump Frequency & $980 \mathrm{~nm}$ \\
\hline Pump power & $100 \mathrm{~mW}$ \\
\hline Polarization Factor & 2 \\
\hline Reyleigh back scattering & $1 / \mathrm{Km}$ \\
\hline
\end{tabular}


TABLE I. HOA PARAMETERS

In star topology with input signal power of $-40 \mathrm{dBm}$, it is observed that at $10 \mathrm{Gbps}$, the numbers of users supported are 5 in number within the acceptable BER range of $2.78569 \mathrm{e}-026$ to $1.14947 \mathrm{e}-009$. At $20 \mathrm{Gbps}$, the number of users supported is 2 in number within the acceptable BER range of 3.2969e-024 to $6.22243 \mathrm{e}-016$. At $30 \mathrm{Gbps}$, star topology is unable to support any user.

In tree topology with input signal power of $-40 \mathrm{dBm}$, it is observed that at $10 \mathrm{Gbps}$, the numbers of users supported are 4 in number within the acceptable BER range of $1.42336 \mathrm{e}-011$ to 3.33672-011. At $20 \mathrm{Gbps}$, the number of users supported is 4 in number within the acceptable BER range of 3.46206e-009 to $3.29799 \mathrm{e}-009$. At $30 \mathrm{Gbps}$, tree topology is unable to support any user.

In mesh topology with input signal power of $-40 \mathrm{dBm}$, it is observed that at $10 \mathrm{Gbps}$, the number of users supported are 8 in number within the acceptable BER range of $1.22645 \mathrm{e}-047$ to $6.70349 \mathrm{e}-015$. At $20 \mathrm{Gbps}$, the number of users supported is 6 in number within the acceptable BER range of 4.55613e-026 to $9.02575 \mathrm{e}-010$. At $30 \mathrm{Gbps}$, mesh topology is unable to support any user.

Mesh topology provides better performance than star and tree topology using Hybrid Raman-EDFA amplifier. In all the three topologies, it is observed that increment in bit rates increases the Bit Error Rate.

\section{v. Conclusion}

This paper concludes that increment in bitrates increases the BER. Mesh Topology provides better results over star and tree topology using Hybrid Raman-EDFA amplifier. Mesh topology at 10Gbps supports 8 users and at $20 \mathrm{Gbps}, 6$ users are supported. Star topology supports 5 users at $10 \mathrm{Gbps}$ and 2 users at $20 \mathrm{Gbps}$. Tree topology supports 4 users at $10 \mathrm{Gbps}$ and $20 \mathrm{Gbps}$. No Users are supported at $30 \mathrm{Gbps}$ in all the three topologies.

\section{Acknowledgment}

The author is thankful to his guide, his god and all the positive energies that helped in the completion of the work

\section{References}

[1] C. Jiang et al., "Improved gain performance of high concentration $\mathrm{Er}_{3+}$ $\mathrm{Yb}_{3+}$ - co doped phosphate fiber amplifier", IEEE J. Quantum Electron. Vol. 41, No. 5, pp. 704, 2005.

[2] M.N. Islam, Raman Amplifiers for Telecommunications-2 Subsystems and Systems, Springer, pp. 430-431, 2004.

[3] R.S. Kaler, "Simulation of $16 \times 10 \mathrm{~Gb} / \mathrm{s}$ WDM system based onoptical amplifiers at different transmission distance and dispersion" Optik 123 , pp. 1654-1658, 2012.

[4] Simranjit Singh, R.S. Kaler, "Placement of hybrid optical amplifierin fiber optical communication systems" Optik 123, pp. 1636-1639, 2012.

[5] Sanjeev Dewra and R.S. Kaler , "Performance evaluation of an optical network based on optical cross add drop multiplexer" J. Opt.Technol. 80 (8) , pp 502- 505, Aug 2013.
[6] Y. N. Singh, V. K. Jain, and H. M. Gupta, "Semiconductor optical amplifiers in WDM star networks," IEE Proc. Optoelectron.,vol. 143, no. 2, pp. 144-152, April 1996.

[7] M. Gerla and L. Fratta, "Tree structured fiber optic MANs," IEEE J. Sel. Areas Commun., vol. 6, no. 6, pp. 934-943, July 1988.

[8] Y. N. Singh, V. K. Jain, and H. M. Gupta, "Semiconductor optical amplifiers in WDM tree-net," J. Lightwave Technol., vol. 15, no. 2, pp. 252-260, Feb. 1997.

[9] J. P. Lang and J. Drake, "Mesh network resiliency using GMPLS,"Proc. IEEE, vol. 90, no. 9, pp. 1559-1564, Sep. 2002.

[10] H. Kobrinski, R. M. Bulley, M. S. Goodman, M. P. Vechi, and C. A. Brackott, "Demonstration of high capacity in the LAMBDANET architecture: multiwavelength optical networks," Electron. Lett., vol. 23, no. 16, pp. 824-826, July 1987.

[11] M. Gerla and L. Fratta, "Tree structured fiber optic MANs," IEEE J. Sel. Areas Commun., vol. 6, no. 6, pp. 934-943, July 1988. 